\title{
High-angle optically accessible Brewster cavity exciton-polaritons
}

\author{
G. Christmann, ${ }^{1}$ A. V. Trifonov, ${ }^{3}$ A. Tzimis, ${ }^{1,6}$ Z. Hatzopoulos,,${ }^{1,4}$ I. V. Iorsh, ${ }^{5}$ J. J. Baumberg, ${ }^{2}$ and P. G. Savvidis ${ }^{1,5,6, *}$ \\ ${ }^{1}$ Foundation for Research and Technology-Hellas, Institute of Electronic Structure and Laser, P.O. Box 1527, 71110 Heraklion, Crete, Greece \\ ${ }^{2}$ NanoPhotonics Centre, Cavendish Laboratory, University of Cambridge, Cambridge CB3 OHE, United Kingdom \\ ${ }^{3}$ Spin Optics Laboratory, Saint Petersburg State University, St. Petersburg 198504, Russia \\ ${ }^{4}$ Department of Physics, University of Crete, 71003 Heraklion, Crete, Greece \\ ${ }^{5}$ Department of Nanophotonics and Metamaterials, ITMO University, 197101 St. Petersburg, Russia \\ ${ }^{6}$ Department of Materials Science and Technology, University of Crete, P.O. Box 2208, 71003 Heraklion, Crete, Greece
}

(Received 28 February 2019; revised manuscript received 11 May 2019; published 3 June 2019)

\begin{abstract}
We report on the observation of the strong-coupling regime between quantum well excitons and a high incidence "Brewster cavity mode" previously identified as the generalized Brewster angle condition in multilayer structures [H. F. Mahlein, J. Opt. Soc. Am. 64, 647 (1974)]. This propagating mode is inside the light cone and therefore can be accessed from the top side of the sample without the need for prism or grating coupling methods. The observed anticrossing is clear evidence of the strong light-matter coupling regime. All the results are accurately reproduced by transfer matrix simulations. These results demonstrate the high potential of such structures for the study of propagating polaritons at high $k$, which could be harnessed for the realization of polaritonic circuit devices.
\end{abstract}

DOI: 10.1103/PhysRevB.99.241402

Resonant optical microcavities are a very active research field as they allow one to tailor the light-matter interaction at optical wavelength scales. Several approaches are used extensively to achieve such optical confinement, e.g., distributed Bragg reflector (DBR) microcavities (MCs), photonic crystals, or microdisks [1]. In particular, planar DBR MCs have been extensively studied in the strong exciton photon coupling regime [2] with the resulting bosonic quasiparticles called polaritons possessing very rich physical properties with promising future device application prospects [3-6]. They exhibit indeed strong $\chi_{3}$ nonlinearity [7] that make them excellent candidates for the realization of integrated circuits. In this context, building blocks such as bistable systems, transistors, and switches have been realized [8-12], with more recent implementations taking advantage of propagating polariton condensates, which can travel distances over $100 \mu \mathrm{m}$ $[13,14]$, required for the realization of circuits.

This idea of propagating polaritons has been further developed with different schemes other than the conventional Fabry-Pérot distributed Bragg mirror (DBR) MC for coupling quantum wells (QWs) with a photonic mode, such as surface Bloch waves [15-18] and optical waveguides [19,20]. These structures present the advantage of requiring fewer growth steps within a single DBR or cladding layer. Furthermore, the mode effective volume is also reduced, increasing the light-matter coupling and thus the Rabi splitting. A drawback of these structures, however, is that the photonic modes are beyond the light cone, therefore they can only be accessed by side coupling, prism coupling, or grating coupling.

In this Rapid Communication, we report the strongcoupling regime (SCR) between QW excitons and the high-

*psav@materials.uoc.gr incident angle photonic mode associated with the generalized Brewster angle condition in multilayer structures [21]. This propagating mode is inside the light cone and therefore can be accessed from the top side of the sample without the need for prism or grating coupling methods. Clear anticrossing is observed with a Rabi splitting of $5 \mathrm{meV}$. Transfer matrix simulations performed on these structures accurately reproduce our experimental results and provide deeper insight into the observed phenomena.

Surface Bloch waves (SBWs) have been known for a long time [22]. They consist of confined waves at the interface between a one-dimensional (1D) quarter-wave stack and a semi-infinite medium of lower refractive index (e.g., air). The original theoretical descriptions studied fully confined eigenmodes in semi-infinite multilayers, and considering solutions with exponential decay along the growth direction, both in air and in the multilayer. These modes are true interface modes and are therefore fully confined in the multilayer with in-plane wave vectors $\left(k_{\|}\right)$beyond the light cone.

This system can be understood qualitatively as a cavity mode in the first DBR layer, confined by the reflection at the dielectric/air interface on one side and by the DBR (minus the first layer) on the other side. With this picture in mind we consider a realistic case with a finite DBR of $<100 \%$ reflectivity and with light at normal incidence. Inside the DBR first layer (which is generally made of the high refractive index material of the multilayer pair), the electromagnetic field has a $0^{\circ}$ reflection phase shift both at the air interface and at the interface with the rest of the DBR at the stopband center. No resonant mode within the stopband is supported by this layer as it is of quarter-wave thickness. For transverse electric (TE) polarization the situation is the same for all in-plane wave vectors $\left(k_{\|}\right)$within the light cone. This absence of modes within the light cone is illustrated in Fig. 1(a) where a TE 

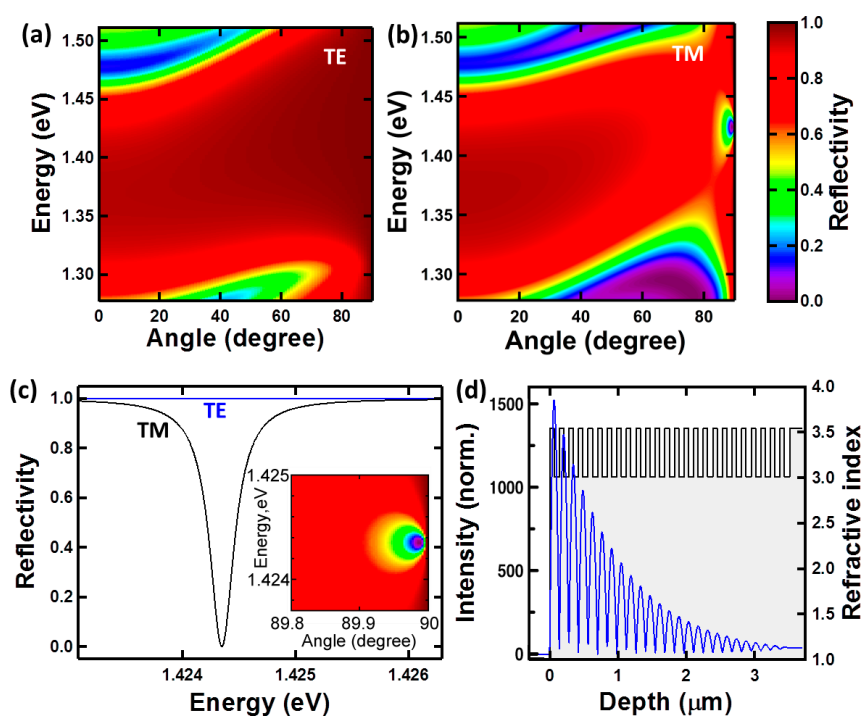

FIG. 1. (a), (b) Calculated angle- and energy-dependent reflectivity images of a ten-pair GaAs/AlAs DBR in TE and TM polarization, respectively. (c) Calculated reflectivity spectra of a 25-pair GaAs/AlAs DBR in TE and TM polarization at $89.99^{\circ}$. The inset is the corresponding reflectivity image close to grazing incidence. (d) Refracting index and corresponding calculated light field intensity profile at resonance.

reflectivity image of a ten-pair GaAs/AlAs DBR is displayed, obtained using transfer matrix simulations [23].

For the transverse magnetic (TM) case the situation is quite different. Within the light cone, when the in-plane wave vector corresponds to an angle past the Brewster angle for the top material/air interface, the amplitude reflectivity coefficient changes its sign, adding a $\pi$ phase shift to the reflection. The first layer now supports a resonant mode at the center of the stopband, which is within the light cone. This mode is generally not easily visible because of the strong imbalance between the high DBR reflectivity and the reflectivity of the first layer/air interface. It is only at the approach of the critical angle $\left(90^{\circ}\right.$ incidence outside) that the first layer/air interface reflectivity becomes equal to that of the DBR, and the mode appears in the middle of the stopband, characterized by a reflectivity going to zero [Fig. 1(b)]. Owing to its similarity with the Brewster angle (TM reflectivity going to zero for a specific angle) this condition has been named the generalized Brewster angle [21]. In the rest of this Rapid Communication, the resonant cavity mode will be referred to as the Brewster cavity (BC) mode.

For $k_{\|}$beyond the light cone, there is total internal reflection at the first layer/air interface and the reflectivity coefficient becomes complex, adding a $k_{\|}$-dependent phase shift. Depending on the value of this phase shift, a mode can then be supported within the first layer. This family of modes beyond the light cone that can be supported by the first DBR layer are the SBWs noted above.

In Figs. 1(a) and 1(b) the number of pairs is kept small in order to easily see the BC mode. For a higher number of layer pairs (25), the BC becomes much sharper [Fig. 1(c)]. The simulated mode exhibits a very narrow linewidth corresponding to a quality factor $\left(Q=\frac{E}{\Delta E}\right)$ of $\sim 5400$ for TM polarization while maintaining high reflectivity in TE. This BC mode also appears only very close to $90^{\circ}$ incidence. Finally, Fig. 1(d) displays the field distribution of this $\mathrm{BC}$ mode, revealing a standing wave strongly confined by the air/DBR interface. There is a node at the air/DBR interface (due to the $\pi$ phase shift) which is then present at every even DBR interface while there is an antinode at every odd DBR interface.

Although SBW and the general Brewster angle condition have been proposed and investigated for quarter-wave layers, one can change the thickness of the first DBR layer and get similar effects. For example, any top layer of thickness $\frac{p \lambda}{2}+\frac{\lambda}{4}$ with $p$ a positive integer will give a BC mode in TM, and conversely $\frac{p \lambda}{2}$ will give a mode in TE. Note that having a TE mode is beneficial, as the DBR reflectivity is higher for this polarization because Fresnel coefficients are larger in TE, and larger $Q$ is therefore expected. As an illustration the TE BC mode corresponding to the structure simulated in Fig. 1(c) with instead a $\frac{\lambda}{2}$ first layer has a simulated $Q$ factor of 34000 instead of $5400\left(\mathrm{BC}\right.$ mode at $\left.89.975^{\circ}\right)$. Note finally that using the low-index material for the top layer will change the phase shift at reflection by $\pi$ on the DBR side and switch the polarization in which the BC mode is observed [21].

We now consider a specific sample in order to explore the possibility of observing the BC mode and eventually achieve strong coupling with it. It consists of an unfolded microcavity in which QWs are incorporated into a Bragg mirror stack [24]. It is made of a stack of quarter-wavelength-thick alternating high refractive index $\left(n_{\mathrm{GaAs}} \sim 3.5\right)$ GaAs layers and of a smaller effective refractive index $\left(n_{\text {eff }} \sim 3.2\right)$ quarterwave pseudolayers of AlAs/GaAs/InGaAs/GaAs/AlAs as displayed in Fig. 2(a). The 10-nm-wide $\mathrm{In}_{0.1} \mathrm{Ga}_{0.9} \mathrm{As} \mathrm{QWs}$ are placed symmetrically inside the pseudolayer. In the current study the measurements are performed at a place on the sample where the exciton energy is close to the center of the DBR stopband at $90^{\circ}$ incidence.

To investigate these $\mathrm{BC}$ modes we use high incidence angle reflectivity [the setup is shown in Fig. 2(b)]. A collimated white light beam from a halogen lamp is focused through a 200-mm focal length lens in a direction parallel to the sample. The position of the sample within the beam is adjusted so that the focal spot is in the sample plane. Because of the large angle of incidence the spot size is quite large and strongly anisotropic $\left(\sim 2 \times 0.2 \mathrm{~mm}^{2}\right)$. Angles very close to $90^{\circ}$ are collected with $\sim 0.5^{\circ}$ angular resolution using a 200-mm focal lens, passed throughout the polarizer and analyzed in a spectrometer. White light reference spectra are acquired to normalize the reflected light. The sample temperature is maintained at $15 \mathrm{~K}$ in a closed cycle He cryostat.

Figure 2(c) displays polarization-dependent reflectivity measurements at a large negative detuning, in order to probe the BC mode alone. As expected, while a photonic mode is clearly observed for the TM polarization, it disappears in the TE polarization, showing that our setup efficiently probes these very high incidence modes. The measured linewidth is $\sim 4.5 \mathrm{meV}$, corresponding to $Q \sim 310$, slightly smaller than the theoretical value of $Q \sim 380$ mainly due to the large size of the probing area and collection over a relatively large angular range.

To investigate the strong light-matter coupling regime of this $\mathrm{BC}$ mode with excitons, we measure the reflectivity 
(a)

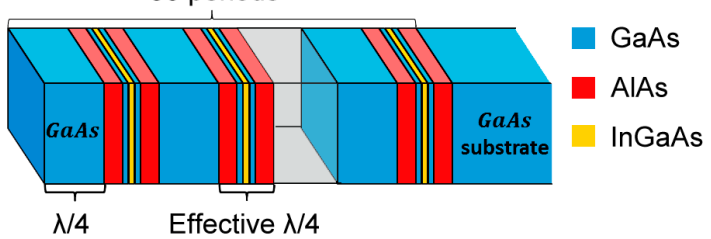

(b)

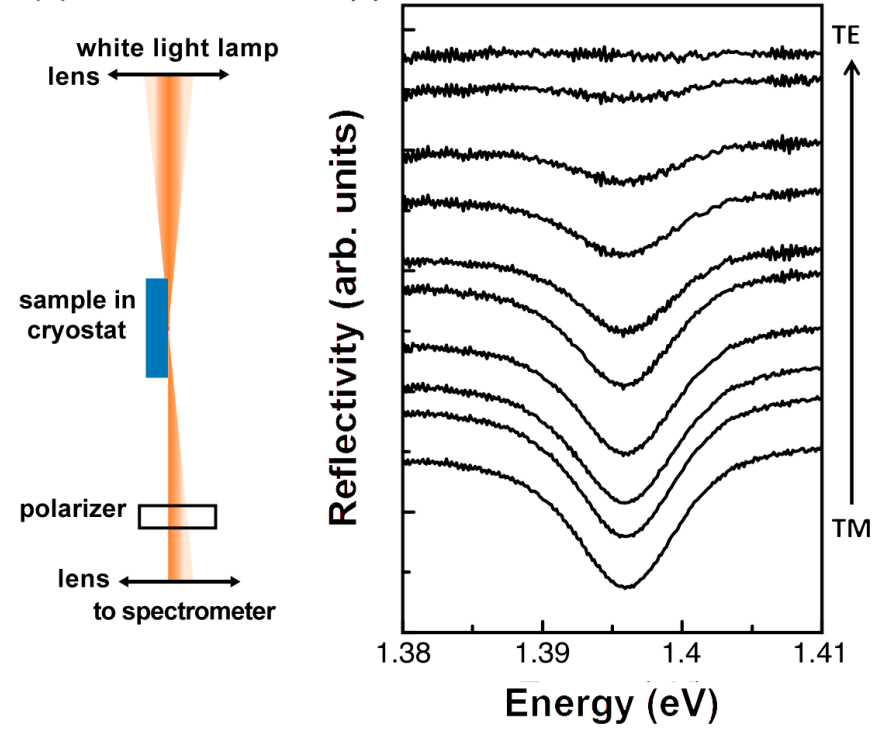

FIG. 2. (a) Schematic of the BC QW sample studied. (b) Schematic of the experimental setup. (c) Polarization-dependent reflectivity measurements at a large negative detuning, spectra taken every $10^{\circ}$ azimuthal polarization at grazing incidence, and shifted for clarity.

spectra as shown in Fig. 3(a), with the spot elongated in the direction of constant exciton-BC mode detuning and scan the sample in the perpendicular direction through the sample wedge with a detuning varying at a rate of $2 \mathrm{meV} / \mathrm{mm}$. Doing this it is possible to tune the Brewster cavity mode through the
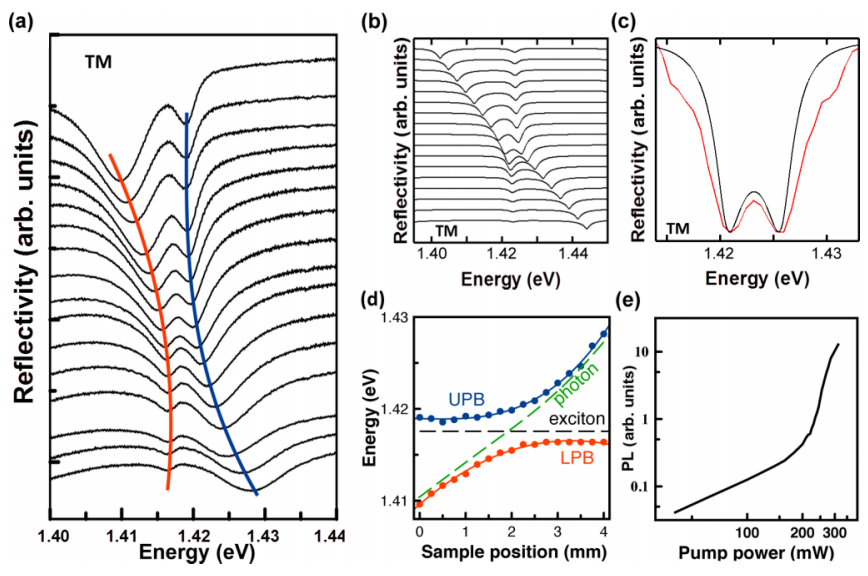

FIG. 3. (a) Position-dependent reflectivity measurements along the sample wedge at grazing incidence. (b) Calculated TM polarized reflectivity spectra at different detuning, shifted for clarity. The angle is integrated between $89^{\circ}$ and $90^{\circ}$. (c) Corresponding dispersion curves vs detuning. (d) Red (gray): Experimental TM reflectivity spectrum at zero detuning. Black: Calculated corresponding reflectivity spectrum. exciton resonance, revealing a clear anticrossing between the lower polariton branch (LPB) and the upper polariton branch (UPB). This demonstrates unambiguously that the system is in the SCR. In Fig. 3(c) the reflectivity dip positions are displayed versus the sample position and the corresponding LPB and UPB are fitted using a two-level model yielding a splitting of $\sim 5 \mathrm{meV}$.

To get a better understanding of the system, we performed transfer matrix simulations of the SCR. The sample is as described in Fig. 2(a) with a Lorentzian resonance [25] used to model the effect of the QWs [Fig. 3(b)]. With this model we are able to reproduce the anticrossing we observed experimentally, as well as the experimental spectrum with a good agreement [Fig. 3(c)]. From the fit, we can extract the value of the Rabi splitting $\left(\Omega_{\mathrm{VRS}} \sim 5 \mathrm{meV}\right)$ which is similar to the splitting previously deduced from the two-level model [Fig. 3(d)].

Clearly mapping of the states in the vicinity of the light cone is crucial to understand the strong-coupling regime observed in our structure. To do this, we perform calculations in a structure with reversed GaAs superstrate and air substrate as shown in Fig. 4(a). In such a hypothetical situation, the light source and detector are located within the GaAs substrate, providing access to this forbidden area of dispersion. In this approach, states outside the light cone [Fig. 4(b)] correspond to the total internal reflectance (TIR) region of $k$ space marked by the bright yellow color. Results for the reflection intensity dependence on the in-plane wave vector $k_{\|}$and energy, without excitons, are shown on Fig. 4(c), with the black dashed line marking the light cone. On the left are photonic states with direct optical access, while those on the right lack direct optical access from the air. The grazing incidence states probed through angle-resolved reflectivity measurements are located on the left of but close to the dashed line, with the BC mode clearly seen (marked by an arrow).

In contrast, the SBW states are outside the light cone and are not seen in the reflected intensity. However, they can be seen from the phase of the reflectance coefficient amplitude, whose dependence on the in-plane wave vector $k_{\|}$and energy is shown in Fig. 4(d) for the same conditions as Fig. 4(c), with the line marked "SBW" corresponding to the SBW dispersion. It is clearly seen that SBW and BC states are connected and transform one into the other, arising from the physical origin of both as localized photonic states, and dependent on their phase shift at the air/structure interface. However, we stress that $\mathrm{BC}$ and SBW are different states, because $\mathrm{BC}$ modes exist inside the light cone while SBW modes are only outside the light cone. The reflection intensity dependence on the in-plane wave vector $k_{\|}$and energy including excitons are shown in Figs. 4(e) and 4(f). Since the strength of the electric field drops with the structure depth and with it, the strength of the exciton-photon coupling, for clarity and to avoid smearing and unnecessary broadening effects from variable coupling strengths, in our model the QWs are placed only in the top ten pairs of the structure. The case of zero detuning between the exciton and BC [Fig. 4(e)] contrasts with that of large negative detuning [Fig. 4(f)]. We note that grazing angle reflectivity measurements probe the dispersions at different in-plane wave vectors. So there is a subtle difference between the anticrossing of dips in the reflectance spectrum shown 

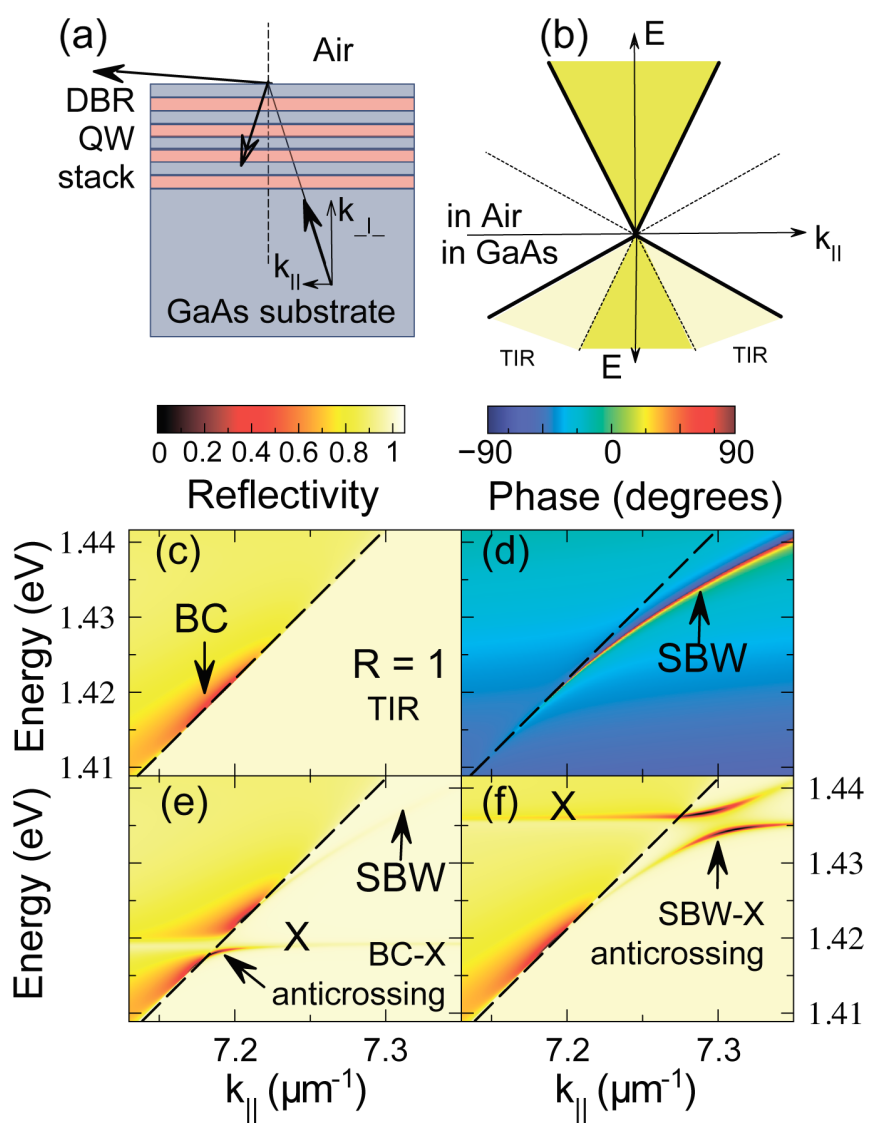

FIG. 4. (a) Light propagation with the light source positioned inside the GaAs substrate allowing access to states beyond the light cone. (b) Light $k$ space in the air (top) and inside the sample (bottom). Solid lines are the light dispersion in air and GaAs, $E=\hbar c k_{\|} / n$. The dark yellow color in (b), (c), (e), and (f) marks optically accessible areas of $k$ space, whereas bright yellow marks "TIR" optically inaccessible states from the air (accessible from the GaAs substrate side). Calculated TM polarization reflectivity plots of (c), (e), (f) amplitude and (d) phase vs in-plane wave vector. The black dashed line marks the light cone borderline. (c) Reflectivity without excitons. Brewster cavity mode marked with BC. (d) Phase of the reflected wave from the structure without excitons. SBW marks the surface Bloch wave. (e) Reflectivity showing SC regime when excitons (X) are resonant with BC. (f) Reflectivity for exciton-BC mode energy detuning of $\delta=-20 \mathrm{meV}$. Modeling parameters were chosen as follows: exciton oscillator strength $\hbar \Gamma_{0}=30 \mu \mathrm{eV}$ and nonradiative broadening $\hbar \Gamma_{\mathrm{NR}}=0.3 \mathrm{meV}$, QWs are placed only in the first ten periods of the structure. in Fig. 3(a) and anticrossing of exciton and hybrid BC-SBW modes living across the light cone.

Finally, we stress that the current structure is not yet optimized for efficient coupling between quantum wells and the Brewster cavity mode. Indeed, as shown in Fig. 1(d), the antinodes of the electric field are located at the interfaces of the different DBR layers while the QWs are in the center of the low-index material layers. This configuration is not optimum because QWs should be ideally located at the standing-wave antinodes, furthermore, quantum wells located deep inside the structure are not well coupled as the electric field decays into the DBR. The insertion of QWs in the low-index material also increases the effective refractive index of the layer, therefore reducing the reflectivity of the DBR and thus the quality factor of the BC mode. Similarly to conventional double DBR MCs, an optimized structure would use a $\frac{p \lambda}{2}$ top layer of the high refractive index with QWs at the antinodes of the electric field. This structure then combines a high $Q$ TE BC mode with efficient coupling to the QWs.

In conclusion, we explored the observation of strong coupling between a BC mode and QW excitons in a Bragg polariton structure. These fully polarized modes appear at a high angle within the light cone at the interface of multilayer structures and their relation to SBWs is confirmed. Clear anticrossing associated with a 5-meV Rabi splitting is observed, revealing the SCR and is accurately reproduced by transfer matrix simulations. Finally, an optimized sample design is proposed allowing for an improved quality factor and efficient coupling with QWs. All these results demonstrate the high potential of these structures for the study of propagating polaritons at high $k_{\|}$which have proved to be very useful for the realization of interferometric [26] and gating [10] devices. Furthermore, similar to SBW and waveguide structures, they require fewer growth steps compared to conventional MCs, with an extra advantage of this high $k_{\|}$mode being within the light cone and being easily accessible.

Financial support from the bilateral Greece-Russia Polisimulator project cofinanced by Greece and the EU Regional Development Fund, Russian Science Foundation Grants No. 19-72-20120, UK EPSRC EP/L027151/1, EP/N016920/1 grants are acknowledged. The research was cofinanced by Greece and EU-ESF Fund through HRDELL 2014-2020 program (project MIS 5004464). G.C. acknowledges fruitful discussions with Dr. M. Kolle. The SPbU resource center "Nanophotonics" is also acknowledged.
[1] K. J. Vahala, Nature (London) 424, 839 (2003).

[2] C. Weisbuch, M. Nishioka, A. Ishikawa, and Y. Arakawa, Phys. Rev. Lett. 69, 3314 (1992).

[3] J. Kasprzak, M. Richard, S. Kundermann, A. Baas, P. Jeambrun, J. M. J. Keeling, F. M. Marchetti, M. H. Szymaska, R. André, J. L. Staehli, V. Savona, P. B. Littlewood, B. Deveaud, and L. S. Dang, Nature (London) 443, 409 (2006); R. Balili, V. Hartwell, D. Snoke, L. Pfeiffer, and K. West, Science 316, 1007 (2007).
[4] T. C. H. Liew, I. A. Shelykh, and G. Malpuech, Physica E 43, 1543 (2011).

[5] S. I. Tsintzos, N. T. Pelekanos, G. Konstantinidis, Z. Hatzopoulos, and P. G. Savvidis, Nature (London) 453, 372 (2008).

[6] A. Dreismann, H. Ohadi, Y. V. I. Redondo, R. Balili, Y. Rubo, S. I. Tsintzos, G. Deligeorgis, Z. Hatzopoulos, P. G. Savvidis, and J. J. Baumberg, Nat. Mater. 15, 1074 (2016). 
[7] P. G. Savvidis, J. J. Baumberg, R. M. Stevenson, M. S. Skolnick, D. M. Whittaker, and J. S. Roberts, Phys. Rev. Lett. 84, 1547 (2000).

[8] A. Amo, T. C. H. Liew, C. Adrados, R. Houdré, E. Giacobino, A. V. Kavokin, and A. Bramati, Nat. Photonics 4, 361 (2010).

[9] R. Cerna, Y. Léger, T. K. Paraïso, M. Wouters, F. MorierGenoud, M. T. Portella-Oberli, and B. Deveaud, Nat. Commun. 4, 2008 (2013).

[10] T. Gao, P. S. Eldridge, T. C. H. Liew, S. I. Tsintzos, G. Stavrinidis, G. Deligeorgis, Z. Hatzopoulos, and P. G. Savvidis, Phys. Rev. B 85, 235102 (2012).

[11] D. Ballarini, M. De Giorgi, E. Cancellieri, R. Houdré, E. Giacobino, R. Cingolani, A. Bramati, G. Gigli, and D. Sanvitto, Nat. Commun. 4, 1778 (2013).

[12] H. S. Nguyen, D. Vishnevsky, C. Sturm, D. Tanese, D. Solnyshkov, E. Galopin, A. Lemaître, I. Sagnes, A. Amo, G. Malpuech, and J. Bloch, Phys. Rev. Lett. 110, 236601 (2013).

[13] E. Wertz, L. Ferrier, D. D. Solnyshkov, R. Johne, D. Sanvitto, A. Lemaître, I. Sagnes, R. Grousson, A. V. Kavokin, P. Senellart, G. Malpuech, and J. Bloch, Nat. Phys. 6, 860 (2010).

[14] G. Christmann, G. Tosi, N. G. Berloff, P. Tsotsis, P. S. Eldridge, Z. Hatzopoulos, P. G. Savvidis, and J. J. Baumberg, Phys. Rev. B 85, 235303 (2012).

[15] M. Liscidini, D. Gerace, D. Sanvitto, and D. Bajoni, Appl. Phys. Lett. 98, 121118 (2011).

[16] S. Pirotta, M. Patrini, M. Liscidini, M. Galli, G. Dacarro, G. Canazza, G. Guizzetti, D. Comoretto, and D. Bajoni, Appl. Phys. Lett. 104, 051111 (2014).
[17] G. Lerario, A. Cannavale, D. Ballarini, L. Dominici, M. De Giorgi, M. Liscidini, D. Gerace, D. Sanvitto, and G. Gigli, Opt. Lett. 39, 2068 (2014).

[18] G. Lerario, D. Ballarini, L. Dominici, A. Fieramosca, A. Cannavale, M. Holwill, A. Kozikov, K. Novoselov, and G. Gigli, Appl. Sci. 7, 1217 (2017).

[19] P. M. Walker, L. Tinkler, M. Durska, D. M. Whittaker, I. J. Luxmoore, B. Royall, D. N. Krizhanovskii, M. S. Skolnick, I. Farrer, and D. A. Ritchie, Appl. Phys. Lett. 102, 012109 (2013).

[20] P. Yu. Shapochkin, M. S. Lozhkin, I. A. Solovev, O. A. Lozhkina, Y. P. Efimov, S. A. Eliseev, V. A. Lovcjus, G. G. Kozlov, A. A. Pervishko, D. N. Krizhanovskii, P. M. Walker, I. A. Shelykh, M. S. Skolnick, and Y. V. Kapitonov, Opt. Lett. 43, 4526 (2018).

[21] H. F. Mahlein, J. Opt. Soc. Am. 64, 647 (1974).

[22] A. Yariv and P. Yeh, Optical Waves in Crystals (Wiley, New York, 2003).

[23] H. A. McLeod, Thin-Film Optical Filters, 2nd ed. (Adam Hilger, Bristol, 1986).

[24] A. Askitopoulos, L. Mouchliadis, I. Iorsh, G. Christmann, J. J. Baumberg, M. A. Kaliteevski, Z. Hatzopoulos, and P. G. Savvidis, Phys. Rev. Lett. 106, 076401 (2011).

[25] E. L. Ivchenko, Optical Spectroscopy of Semiconductor Nanostructures (Springer, Berlin, 2004), p. 437.

[26] G. G. Paschos, T. C. H. Liew, Z. Hatzopoulos, A. V. Kavokin, P. G. Savvidis, and G. Deligeorgis, Sci. Rep. 8, 10092 (2018). 